\title{
Participación comunitaria en el mecanismo Transferencias Directas Condicionadas del Programa Bosques
}

\section{Grace Palacios}

https://orcid.org/0000-0003-0892-9036

Universidad de Florida gmpalacios@ufl.edu

\section{RESUMEN}

Chirikyacu, Chunchiwi y Chirik Sacha, ubicadas en la región San Martín (Perú), son comunidades nativas afiliadas al Programa Bosques. En las tres se aprobó la implementación del mecanismo Transferencias Directas Condicionadas (TDC) como instrumento financiero para la conservación de su bosque primario. Esta investigación caracteriza la participación de los beneficiarios a partir del tipo de involucramiento y cantidad de participantes en la ejecución del TDC, identificando factores que facilitan o limitan la participación del grupo beneficiario minoritario.

Las minorías, como en cualquier sociedad, acceden a menores oportunidades. En las tres comunidades se encontraron grupos minoritarios más numerosos (e.g.: mujeres) que otros (e.g.: monolingües), también se encontraron normas de género aceptadas que limitan a los grupos minoritarios de experimentar desenvolvimiento y aprendizaje. Con los aportes del $86 \%$ de familias beneficiarias, se identificó un sistema no complementario de género en la mayoría de las actividades del programa, que además es predominante en el grupo más favorecido de la comunidad.

Se concluye que el grupo minoritario principal puede aumentar la cantidad de participantes a partir de las oportunidades que permita la complementariedad de género que rige en cada comunidad 
(factor interno) y del enfoque de género que incorpore el programa Bosques (factor externo).

Palabras clave: participación comunitaria, complementariedad de género, comunidades indígenas, incentivos económicos para conservación, San Martín, Amazonía peruana

\title{
Community Participation in the Conditional Direct Transfer Mecha- nism of the Bosques Program
}

\author{
ABSTRACT
}

Chirikyacu, Chunchiwi, and Chirik Sacha, located in the San Martin region (Peru), are indigenous communities affiliated with the National Program of Forest Conservation for Climate Change Mitigation. The three communities approved the implementation of the Conditional Direct Transfer Mechanism (TDC, acronym in Spanish) as a financial tool for primary forest conservation. This research characterizes the participation of the beneficiaries from the type of involvement and participation size along the TDC execution, identifying drivers that facilitate and limit the participation of the beneficiaries 'minority.

Minorities, as in any society, have access to fewer opportunities. In the three communities, I found larger minority groups (such as women) than others with less population (such as monolingual), as well as gender norms accepted in the communities, which limit the minority groups from personally growing and learning. With the inputs of $86 \%$ of the Program's beneficiary families, I identified a non-complimentary gender system in most of the program's activities, which is also dominant in the most favored group in the communities.

In conclusion, the major minority group can increase the participants size, from the opportunities that allow the gender complementarity current in each community (internal driver) and the gender approach that the Program incorporates (external driver).

Keywords: Community Participation, Gender Complementarity, Indigenous Communities, Economic Incentives for Conservation, San Martín, Peruvian Amazon 


\section{INTRODUCCIÓN}

En el año 2010, el Ministerio del Ambiente lanzó el Programa Nacional de Conservación de Bosques para la Mitigación del Cambio Climático con el fin de conservar 54 millones de hectáreas de bosques tropicales en diecisiete regiones, $42 \%$ del territorio peruano. Este programa se ejecuta a través de un convenio entre el Estado y las comunidades nativas, comprometiéndolas a conservar bosque primario a cambio de recibir incentivos económicos con condiciones de uso por un periodo determinado (inicialmente de cinco años). Asimismo, el Estado se compromete a acompañar a las comunidades en todo el proceso de implementación del mecanismo de Transferencias Directas Condicionadas (TDC), brindando capacitación, monitoreo y asistencia técnica (Bosques, 2016).

Para la ejecución de las TDC, se deben cumplir seis pasos: (i) focalización; (ii) admisión; (iii) afiliación; (iv) liquidación de transferencias; (v) cumplimiento de condicionalidades, y (iv) graduación. Esta investigación se enfoca únicamente en aquellos en los que la comunidad está involucrada (2, 3, 4 y 5), con mayor énfasis en el último, cumplimiento de condicionalidades, que es la ejecución del plan de inversión que condiciona el uso de los fondos recibidos para la conservación de hectáreas de bosque. Note que una de las metas principales para las comunidades es lograr el cumplimiento del Plan de Inversión, cubriendo cuatro componentes (social, gestión, económico y ambiental).

Entre las comunidades nativas que se afiliaron, a inicios del programa, en la región San Martín, se encuentran Chirik Sacha, Chirikyacu y Chunchiwi, en ese mismo orden de afiliación (por lo tanto, de las tres, Chirik Sacha es la que más tiempo tiene en el programa). Chirikyacu y Chunchiwi están ubicadas en el distrito de San Roque de Cumbaza, en la parte central de la provincia de Lamas; Chirik Sacha, en el distrito San José de Sisa, en la provincia de El Dorado, a pesar de que la mayor parte de su territorio está ubicado en el distrito de Alto Saposoa 
(provincia de Huallaga). Las tres son comunidades indígenas kichwa, con más de diez años de experiencia trabajando con programas de desarrollo rural ${ }^{1}$.

Cabe destacar que la extracción recursos maderables incrementa la economía familiar y reduce la pobreza; se trata de una salida lucrativa inmediata, pero perjudicial a largo plazo, porque conlleva a la explotación del recurso y a incrementar los índices de pobreza para la zona (Ocaña, 2012). Con la propuesta de conservación de hectáreas del Programa Bosques, al 2017 Chirikyacu tiene 4150 hectáreas y de incentivo recibe S/ 41 500; Chunchiwi, 6200 hectáreas y recibe S/ 62 000, y Chirik Sacha, 1899 hectáreas y recibe S/ 18990.

Cada comunidad está liderada por la Junta Directiva, que se renueva cada dos años, y para una mejor administración comunal, se organizan por comités. Los miembros de la comunidad voluntariamente se postulan a cualquiera de los cargos de la Junta. A veces, exmiembros de la Junta recomiendan miembros para el siguiente periodo. Las Juntas Directivas y la mayoría de los comités están compuestos por el apu (presidente - jefe comunal), vicepresidente, secretario, tesorero y dos vocales. Las decisiones se toman por mayoría comunitaria, en reuniones donde (idealmente) asiste un representante por comité y uno por familia (en su gran mayoría, varones).

Se estima que, en la Amazonía, las zonas con mayor cobertura de bosque son las que más presentan desigualdad de género (Minam, 2016), lo que se convierte en una desventaja para el desarrollo de las poblaciones amazónicas que trabajan conservando hectáreas de bosque. En el caso de estas tres comunidades, para atender las inequidades de género, las mujeres deben tener una posición más consolidada y representativa en la toma de decisiones de sus comunidades y territorios. En una categoría binaria de cualquier sociedad, «las áreas de autonomía, autoridad y poder, pueden variar» (Gero y Scattolin, 2002) porque no es determinado por un solo género. Por lo tanto, en una categoría binaria, hay diferentes sistemas de género: complementario, jerárquico y patriarcal, por listar algunos ejemplos (Gero y Scattolin, 2002). La creación de comités representativos es un buen paso inicial para el empoderamiento social, cultural y político (Schmink y García, 2015). No obstante, se enfrentan otro tipo de adversidades que se detallan más adelante, en el documento.

1 En las entrevistas, varios productores de cacao en Chunchiwi y Chirik Sacha mencionaron haber participado en el Programa de Desarrollo Alternativo (PDA) de USAID, incorporando el cultivo de cacao entre 2006 y 2010. Chunchiwi, Chirik Sacha y Chirikyacu mencionaron también a otras instituciones con otros programas no relacionados con el cacao en esos mismos años. 
Entre los comités organizados están el Comité de Turismo (Chirikyacu), el Comité de Sacha Inchi (Chirikyacu), y el Comité de Vigilancia y Comité de Artesanía (o de mujeres artesanas). Estos dos últimos fueron organizados a partir del contrato con el Programa Bosques, y están vigentes en las tres comunidades.

Para evaluar la participación del varón y de la mujer en estos comités, se deben entender los roles de género desde una perspectiva sistémica (Paulson, 2000), considerando que en cada sociedad se han adaptado o desarrollado diferentes normas de género que han evolucionado en el tiempo y que son influenciadas por un sinfín de patrones de intersección (Van, 2000; Paulson, 2000). Consecuentemente, los resultados de esta investigación no son una definición permanente porque los sistemas de género cambian y están en constante reconstrucción (Van, 2000).

Así, para definir la participación, esta investigación parte desde el sistema de complementariedad de género, que refiere a la coexistencia (binaria) de ambos (Gero y Scattolin, 2002; Van, 2000), y considera al involucramiento directo e indirecto, según cantidad de participantes (alto, medio, bajo) en cada actividad del plan de inversión.

\section{OBJETIVO}

Caracterizar la participación de los comuneros y comuneras beneficiarios, en las actividades para la implementación del mecanismo TDC.

\section{MÉTODOS, MUESTRA Y ENFOQUE}

Inicié este proyecto identificando todas las actividades en la implementación del mecanismo TDC. Lo realicé a través de la revisión de documentos existentes y entrevistas semiestructuradas. Las entrevistas se realizaron a través de observación participativa y grupos focales mixtos.

Analicé los resultados de la revisión bibliográfica a partir de los documentos elaborados por Bosques-San Martín a lo largo de la intervención del proyecto (reportes de avance) y entrevistas semiestructuradas para identificar los cambios en la comunidad. También realicé talleres participativos que me permitieron validar todos los datos recopilados, junto con conversaciones informales con el equipo de Bosques-San Martín. 
Lista de métodos empleados:

- Encuestas grupales (solo Chirikyacu y Chunchiwi)

- Encuestas individuales

- Encuestas individuales después de las entrevistas sobre las actividades del programa

- Entrevistas semiestruturadas

- Grupo focal (solo Chirik Sacha)

- Taller de validación (solo Chirikyacu y Chunchiwi)

- Visita a parcelas de cacao

- Conversaciones informales con equipo Bosques-San Martín

La recepción en las tres comunidades fue altamente grata, y la disposición de cada comunero y comunera, también. Así, se logró la contribución del $86 \%$ de las familias beneficiarias de artesanía y cacao: en Chirikyacu, 88\%; en Chunchiwi, $86 \%$, y en Chirik Sacha, $85 \%$ (tabla 1-1). La muestra es por familia, pues por cada una hay un beneficiario principal. Hay algunas familias que tienen dos beneficiarios principales, y en ese caso cada uno corresponde a distintos comités. Cabe destacar que el impacto directo del programa es por individuo y no por familia.

En la complementariedad de género, hay dependencias colaborativas para completar el trabajo, lo cual es un atributo de participación mutua (Gero y Scattolin, 2002), también definida como interdependencia múltiple (Paulson, 2000). Estas se basan en enfoques diferenciados al integrar al individuo interdependiente en diferentes niveles. De esta forma, sus roles son partes integrales de igual importancia en el proceso de concretar una actividad (Gero y Scattolin, 2002; Paulson 2000). Del mismo modo, Ruedas (2003), en su observación a McCallum (2001), destaca la importancia sobre el constante cambio en los roles de género en función de las actividades diarias entre grupos cashinahua (grupo indígena amazónico). Ruedas concluye que no se puede evaluar desde una postura jerárquica, sino permitir el protagonismo a los roles en las diferentes actividades.

Por lo tanto, esta investigación caracteriza la participación por tipo de involucramiento (coloración en tabla 1-2) y cantidad de participantes (escalas en tabla 1-2), y reconoce tres sistemas de complementariedad de género a partir de una categoría binaria (tabla 1-4):

- Tipo de involucramiento: directo (azul en tabla 1-2) /indirecto (blanco en tabla $1-2)$

- Escala según cantidad de participantes: alto/medio/bajo

- Sistemas de complementariedad: complementario y no complementario 
Tabla 1-1. Total de comuneros y comuneras que participaron en levantamiento de información

\begin{tabular}{lccccc}
\hline $\begin{array}{l}\text { Comunidad nativa } \\
\text { (población total) }^{*}\end{array}$ & $\begin{array}{c}\text { Solo artesanas / } \\
\text { Total } \\
\text { artesanas }^{* * *}\end{array}$ & $\begin{array}{c}\text { Artesanas } \\
\text { y a la vez } \\
\text { cacaoteros }\end{array}$ & $\begin{array}{c}\text { Solo cacaoteros / } \\
\text { Total } \\
\text { cacaoteros }\end{array}$ & Otro $^{* * *}$ & Total** $^{*}$ \\
\hline Chirikyacu (26) & $5 / 13$ & $8 / 8$ & $10 / 21$ & $3 / \mathrm{X}$ & $\mathbf{2 3 / 2 6}$ \\
Chunchiwi (62) & $2 / 10$ & $8 / 9$ & $14 / 27$ & $\mathrm{X}$ & $\mathbf{2 4 / 2 8}$ \\
Chirik Sacha (56) & $10 / 16$ & $3 / 3$ & $9 / 13$ & $2 / \mathrm{X}$ & $\mathbf{2 2 / 2 6}$ \\
\hline
\end{tabular}

* Se obtuvo de las actas del convenio llenadas por la Junta Directiva en su primer año en el mecanismo TDC.

** Beneficiarios del mecanismo, pero no de artesanía ni de cacao. Son los involucrados en otros comités, como Turismo, en el caso de Chirikyacu, u otras capacitaciones, como GPS y actividades de patrullaje. No se suma al total.

*** Se obtuvo de las listas provistas por sus respectivos promotores o técnicos y presidentes de los comités de artesanía/mujeres organizadas.

En las tablas 1-2 y 1-3, el tipo de involucramiento directo se distingue porque se muestra en azul, mientras que el indirecto, blanco. Para identificar cuándo es directo e indirecto, se consideraron los sistemas de género complementarios y no complementarios, identificando tres: mutuo e integrador (ambos, complementarios) y no complementario (tabla 1-4).

Por lo tanto, es involucramiento directo cuando el sistema de complementariedad es mutuo y también cuando es no complementario. Cuando es integrador, puede ser tanto directo como indirecto, dependiendo de quién lidere, si el varón o la mujer (tabla 1-4).

\section{HALLAZGOS PRINCIPALES}

Las normas de género son construidas socialmente en sus roles. Estas son aprendidas a una escala muy local — en este caso, comunitaria — y son «enseñadas a través de normas culturales vigentes, limitaciones y consejos» (Gero, y Scattolin, 2002). Las normas de género pueden crear diferencias y dependencias (Gero, y Scattolin, 2002; Paulson, 2000; Van, 2000), y es a partir de estas que se identificó a los grupos minoritarios dentro de la comunidad. Se trata de minorías, porque tienen menos poder de decisión y de participación dentro de la comunidad. 
Tabla 1-2. Tipo de involucramiento por cantidad de participantes mujeres

\begin{tabular}{|c|c|c|c|}
\hline Escala & Chirikyacu & Chunchiwi & Chirik Sacha \\
\hline \multirow{7}{*}{$\stackrel{\circ}{\stackrel{ }{4}}$} & Actividades Vivero & Capacitación artesanías & Actividades vivero \\
\hline & $\begin{array}{l}\text { Comercialización } \\
\text { artesanías }\end{array}$ & Cosecha de cacao & $\begin{array}{l}\text { Comercialización } \\
\text { artesanías }\end{array}$ \\
\hline & Plantones a la chacra & Plantación cacao & Residuos sólidos \\
\hline & & Saberes ancestrales & Biohuerto \\
\hline & & Vigilancia bosques & Cuyes \\
\hline & & & Asociatividad comunal \\
\hline & & & Compostaje \\
\hline \multirow{7}{*}{ 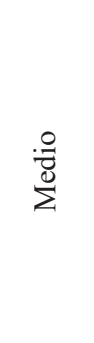 } & Asociatividad comunal & Asociatividad comunal & GPS y brújulas \\
\hline & Planificación & Planificación & Vigilancia de bosques \\
\hline & Rendición de cuentas & Actividades en el vivero & Saberes ancestrales \\
\hline & Talleres agroforestales & Talleres agroforestales & Cosechas de cacao \\
\hline & & Abonamiento de suelo & \\
\hline & & Recibos de pago & \\
\hline & & Organización y gobernanza & \\
\hline \multirow{8}{*}{$\stackrel{\circ}{\frac{\pi}{\pi}}$} & Recibos de pago & Rendición de cuentas & Organización y gobernanza \\
\hline & GPS y brújulas & GPS y brújulas & Características de su suelo \\
\hline & Transporte de Bokashi & Monitoreo plan de vida & $\begin{array}{l}\text { Negocios y } \\
\text { comercialización de otros } \\
\text { productos }\end{array}$ \\
\hline & Elaboración de Bokashi & $\begin{array}{l}\text { Implementación cocina } \\
\text { communal }\end{array}$ & Bokashi \\
\hline & $\begin{array}{l}\text { Acompañamiento de } \\
\text { Técnico a parcela }\end{array}$ & $\begin{array}{l}\text { Legislación, delitos e } \\
\text { infracciones }\end{array}$ & $\begin{array}{l}\text { Acompañamiento de } \\
\text { técnico a parcela }\end{array}$ \\
\hline & Vigilancia de bosques & & \\
\hline & Saberes ancestrales & & \\
\hline & Manejo de residuos sólidos & & \\
\hline
\end{tabular}


Tabla 1-3. Tipo de involucramiento directo (azul) e indirecto (incoloro) del varón

\begin{tabular}{|c|c|c|}
\hline Chirikyacu & Chunchiwi & Chirik Sacha \\
\hline Actividades vivero & Actividades vivero & Actividades vivero \\
\hline Asociatividad comunal & Asociatividad comunal & Asociatividad comunal \\
\hline Vigilancia de bosques & Vigilancia bosques & Vigilancia de bosques \\
\hline GPS y brújulas & GPS y brújulas & GPS y brújulas \\
\hline Saberes ancestrales & Saberes ancestrales & Saberes ancestrales \\
\hline $\begin{array}{l}\text { Acompañamiento de } \\
\text { técnico a parcela }\end{array}$ & Abonamiento de suelo & $\begin{array}{l}\text { Acompañamiento de } \\
\text { técnico a parcela }\end{array}$ \\
\hline Talleres agroforestales & Talleres agroforestales & $\begin{array}{l}\text { Negocios y } \\
\text { comercialización de otros } \\
\text { productos }\end{array}$ \\
\hline Plantación de cacao & Plantación de cacao & $\begin{array}{l}\text { Comercialización de } \\
\text { artesanías }\end{array}$ \\
\hline Transporte de Bokashi & Cosecha de cacao & Cosecha de cacao \\
\hline Elaboración de Bokashi & $\begin{array}{l}\text { Implementación de cocina } \\
\text { comunal }\end{array}$ & Elaboración de Bokashi \\
\hline Rendición de cuentas* & Rendición de cuentas* & Compostaje \\
\hline Residuos sólidos & Monitoreo plan de vida & Residuos sólidos \\
\hline $\begin{array}{l}\text { Comercialización de } \\
\text { artesanías }\end{array}$ & $\begin{array}{l}\text { Organización indígena y } \\
\text { gobernanza* }\end{array}$ & $\begin{array}{l}\text { Organización indígena y } \\
\text { gobernanza* }\end{array}$ \\
\hline Planificación* & Planificación* & Características de su suelo \\
\hline \multirow[t]{3}{*}{ Recibos de pago* } & Recibos de pago* & Biohuerto \\
\hline & Capacitación artesanías & Cuyes \\
\hline & $\begin{array}{l}\text { Legislación, delitos e } \\
\text { infracciones * }\end{array}$ & \\
\hline
\end{tabular}

*Actividades solo para la Junta Directiva o el Comité de Gestión. Solo un grupo selecto de varones tuvo involucramiento directo. 
Tabla 1-4. Sistemas de complementariedad de género

\begin{tabular}{|c|c|c|c|}
\hline Sistema & Definición & Descripción & Actividades principales \\
\hline \multirow{4}{*}{$\begin{array}{l}\text { No } \\
\text { complementaria }\end{array}$} & \multirow{4}{*}{$\begin{array}{l}\text { Trabaja } \\
\text { individualmente } \\
\text { sin integrar al otro } \\
\text { individuo }\end{array}$} & $\hat{\sigma} \neq q$ & Talleres agroforestales \\
\hline & & Trabajo individual & $\begin{array}{l}\text { Saberes ancestrales } \\
\text { (quechua)** }\end{array}$ \\
\hline & & & $\begin{array}{l}\text { Acompañamiento } \\
\text { de técnico a parcela } \\
\text { durante su visita }\end{array}$ \\
\hline & & & GPS y brújulas*** \\
\hline \multirow{4}{*}{ Integrador* } & \multirow{4}{*}{$\begin{array}{l}\text { Uno lidera } \\
\text { integrando al otro } \\
\text { individuo. Las } \\
\text { normas de género } \\
\text { están definidas. }\end{array}$} & $\hat{\sigma} \rightarrow$ ㅇ & \\
\hline & & Varón apoya a mujer & \\
\hline & & $\hat{\sigma} \leftarrow q$ & Vigilancia \\
\hline & & $\begin{array}{l}\text { Mujer apoya al } \\
\text { varón }\end{array}$ & \\
\hline \multirow{4}{*}{ Mutuo } & \multirow{4}{*}{$\begin{array}{l}\text { Hay colaboración } \\
\text { mutual donde } \\
\text { cualquiera puede } \\
\text { liderar. Los } \\
\text { roles no están } \\
\text { prescritos. }\end{array}$} & $\hat{\jmath} \leftrightarrow q$ & Actividades del vivero \\
\hline & & $\begin{array}{l}\text { Los dos se apoyan } \\
\text { mutuamente }\end{array}$ & $\begin{array}{l}\text { Cuidado de su chacra } \\
\text { familiar }\end{array}$ \\
\hline & & & $\begin{array}{l}\text { Transporte de } \\
\text { plantones de cacao }\end{array}$ \\
\hline & & & Cosecha de cacao \\
\hline
\end{tabular}

*Solo muestra a la mujer apoyando al varón, porque no se registraron actividades representativas del varón apoyando a la mujer, coincidente en las tres comunidades.

** La interacción es directamente con los niños, ambos padres son motores de motivación. Esta es la única actividad que se puede retirar de la lista

*** En Chirik Sacha se registró a mujeres que recibieron la capacitación; no obstante, no significa que hubo colaboración mutua para el aprendizaje de esta actividad. 
Las diferencias y dependencias pueden ser tanto intergeneracionales como intergenéricas y pueden ser regidas por edad, grupo, sexo, tiempo, espacio y otras identidades de género (Gero y Scattolin, 2002; Paulson, 2000; Van, 2000). En el caso de las tres comunidades, se han identificado cinco minorías, la más numerosa es la que corresponde a «varón-mujer».

A continuación, listo las cinco minorías como: «individuo con mayor oportunidad $\rightarrow$ individuo discriminado, con menor oportunidad».

- Varón $\rightarrow$ mujer

- Joven $\rightarrow$ adulto mayor

- Alfabetizado $\rightarrow$ analfabeto

- Escolarizado $\rightarrow$ no escolarizado

- Bilingüe $\rightarrow$ monolingüe (quechua hablante).

La desigualdad de género en la Amazonia peruana gira en torno a seis factores, entre ellos, la deserción escolar (Pinedo, 2014; USAID, 2014; Schmink y García, 2015). Son más los varones que concluyen los estudios secundarios, lo que justifica los índices de analfabetismo y el nulo o bajo nivel del dominio del castellano en las mujeres (USAID, 2014). Los factores restantes son la falta del Documento Nacional de Identidad, la violencia doméstica, falta de servicios de salud (USAID, 2014; Schmink y García, 2015, en su cita a Piqué, 2005), falta de capacidad de los gobiernos para integrarlas y falta de enfoque de género (Pinedo, 2014; Avilez, 2008). Los actores que trabajan con comunidades indígenas deben tomar en cuenta estos factores desde la formulación de sus programas para reducir la brecha en la desigualdad de género, que según Ocaña (2012) en su cita a Cavendish (1999), se podría reducir hasta un $30 \%$ si hay un incremento en la economía familiar.

\section{Involucramiento de beneficiarios en los pasos 2-5 del mecanismo TDC}

La participación de los comuneros en el paso 2 se resume en el involucramiento en las reuniones informativas para decidir si afiliarse al programa o no; en el paso 3 , en el involucramiento en las reuniones sobre la firma del convenio, planes de vida e inversión.

En Chunchiwi, asistir es un compromiso comunal, aun cuando no haya un interés personal. La gran mayoría de mujeres y otros pobladores no recuerda los detalles de estas reuniones. En la lista de asistencia, ni en Chunchiwi ni en Chirik Sacha figura la asistencia de las mujeres: solo se evidencia su asistencia a través del registro fotográfico y testimonios orales. No firmaron la ficha de asistencia 
porque lo hace el esposo, o porque firman con el nombre de sus esposos para evitar multas. En este caso ocurre lo mismo que señala Avilés (2008) en su experiencia con mujeres waorani: que puedan asistir no significa que tengan liderazgo o una posición influyente.

En las tres comunidades el número de asistentes a la primera reunión con el programa fue el más alto, y el porcentaje de la asistencia de mujeres se mantuvo igual en Chirikyacu y Chunchiwi. No obstante, a pesar de que Chunchiwi tuvo mayor asistencia de mujeres en las reuniones iniciales, tiene menor presencia de ellas en el cumplimiento de condicionalidades (paso 5).

La mayoría de los comuneros no recuerda estas reuniones en detalle, con excepción de quienes fueron miembros de las Juntas Directivas y Comités de Gestión. No obstante, cada comunidad beneficiaria diseña su plan de inversión, y cubre cuatro componentes con diferentes actividades (varía por comunidad, y algunas actividades son obligatorias). Las actividades no son las mismas en las comunidades, hay algunas excepciones (e.g.: turismo en Chirikyacu, cuyes en Chirik Sacha).

Mientras las comunidades de San Martín fueron avanzando en sus contratos, Bosques-Lima exigió priorizar las actividades del componente económico. De este modo, empezando el segundo a tercer año del contrato, las actividades sociales se redujeron. Cada comunidad decidió qué actividades del componente social descartarían. Entre estas, destaca «Saberes ancestrales para los niños» ${ }^{2}$. Las actividades impulsadas del componente económico fueron cacao y artesanía. Se tomó esta decisión desde Lima, con el fin de prepararlos para cuando salieran del mecanismo TDC y dejasen de recibir los incentivos económicos ${ }^{3}$.

La participación de los comuneros en el paso 4, «Liquidación de transferencias», se resume en las transacciones para recibir fondos (que corresponden solo a la Junta Directiva, formada por varones) y las reuniones de rendiciones de cuentas del uso de fondos en la comunidad. Algunas mujeres recuerdan haber asistido a estas reuniones, pero ninguna hizo preguntas de algo que no entendiera o con lo que estuviera en desacuerdo. La máxima participación de mujeres en este paso es la de las mujeres del Comité de Artesanía, quienes ahora reciben el dinero (a través de la Junta Directiva) para los gastos de su comité.

2 «Saberes ancestrales» era un espacio para estimular el conocimiento del quechua a través de la identidad cultural, dirigido a todos los niños de la comunidad. Era liderado por un comunero que la comunidad reconocía como indicado. No siempre era la misma persona: a veces también participada alguna comunera u otro comunero.

3 Información recopilada durante las conversaciones informales con el equipo del Programa Bosques-San Martín. 
Los comités de gestión se crearon en las primeras comunidades afiliadas al programa. Sin embargo, en Chirik Sacha el Comité de Gestión duró menos de tres años, y en Chirikyacu y Chunchiwi, solo un periodo. Los comités de gestión generaron conflicto en la organización comunal, porque fue un requerimiento externo (no propio de la comunidad) para administrar intereses comunales (como el dinero), de lo que la Junta Directiva es responsable. Chunchiwi es una de las comunidades que pudo gestionar su plan de inversión exitosamente una vez disuelto el Comité de Gestión.

En cuanto al involucramiento de la mujer en las actividades del Plan de Inversión, es mayormente indirecto: cumplen un rol secundario. Esto se da en las tres comunidades, a pesar de las variaciones en la cantidad de mujeres participantes y de las varias actividades disponibles por comunidad (tabla 1-2).

Por cómo se plantearon las preguntas en las entrevistas, la mujer parecía no tener participación alguna. Por ello se reformularon: para indagar más sobre el rol colaborativo que tiene en actividades concretas en las cuales sus esposos también participan. El involucramiento indirecto de la mujer puede ser tan sencillo como recordar a su esposo que asista sus reuniones, o tan significativo como prepararle la comida para ir a patrullar: «le hago fiambre, le alisto sus cosas, le preparo con lo que necesita para la lluvia, fósforos, cigarro, poncho, armas, machete, también chicha como para dos a tres días» (anónimo, CCNN Chirikyacu); «le preparo su fiambre, su tazón y su cuchara, si va a llevar su ollita, para que lleve, tiene una taza. Si hay huevo, huevo se le cocina, sino su ají pucunucho se le da, jaja» (anónimo, CCNN Chirikyacu).

Hay actividades que requieren más individuos, como los talleres agroforestales, y otras que se pueden realizar individualmente, como acompañar al técnico a la parcela de cacao. Cuando la actividad requiere más de un individuo, es colaborativa. Para las mujeres, la artesanía y las actividades del vivero (plantones de cacao) son las más colaborativas y son también sus espacios de mayor aprendizaje. Esta situación se refleja en las tres comunidades.

La cantidad de mujeres participantes (ver escala en tabla 1-2) responde al número de años del TDC en la comunidad (Chirik Sacha es la que más años tiene en el mecanismo). También es notorio que, a mayor número de participantes, mayor involucramiento directo (ver azules en escala alta, tabla 1-2).

En cuanto al involucramiento de los varones, es directo en la mayoría de las actividades del Plan de Inversión. Hay muy pocas actividades de involucramiento indirecto, como en artesanía, o biohuerto (ver tabla 1-3). 
Aunque en las tres comunidades asista el $100 \%$ de los beneficiarios a los talleres de capacitación o a las reuniones, el conocimiento del varón respecto del programa y su aprendizaje del cultivo de cacao es superior que el de la mujer. En cuanto a organización y administración, es notorio el conocimiento en aquellos varones que fueron miembros del Comité de Gestión y la Junta Directiva desde los primeros años de convenio, ya que son quienes más capacitaciones recibieron dentro y fuera de la comunidad (ver asteriscos en tabla 1-3).

La tabla 1-3 muestra que Chirik Sacha es la comunidad donde los varones tienen más actividades de involucramiento indirecto, es decir, es la comunidad donde más mujeres tuvieron involucramiento directo (tabla 1-2). Esto se debe a la variedad de opciones disponibles para las mujeres (biohuertos, crianza de animales menores, residuos sólidos y artesanía). De este modo, los varones apoyaron a sus esposas cuando ellas lo solicitaron (en pocos casos). Se distingue que hay algunas actividades de involucramiento directo, que corresponden a un grupo reducido de varones, como recibos de pago ${ }^{4}$ (ver asteriscos en la tabla 1-3). Esto no ocurre con la mujer, excepto por la liquidación de transferencias para el comité de artesanas.

El involucramiento indirecto también es significativo, porque corresponde a un sistema de complementariedad integrador. En el siguiente ejemplo, las mujeres lideran la actividad de ropa para venta (comercialización de artesanías). Ella sabe que su esposo no sabrá cómo, y está dispuesta a enseñarle ${ }^{5}$ : «mi esposo siempre pregunta "para qué es un vestido" o "qué va con el otro vestido", porque quiere saber lo que necesitamos para que él vaya a traer los vestidos. Nosotros le tenemos que indicar el tipo de vestido que debe traer, él no sabe» (anónimo, CN Chirikyacu).

Cada año de convenio, las actividades en el Plan de Inversión se modifican según las solicitudes de la comunidad y los componentes que haga falta impulsar según el enfoque del programa. Por lo tanto, las comunidades cuentan o contaron con algunas actividades únicas, por decisión comunal. Por ejemplo, Chirik Sacha con los servicios del registrador comunal, Chirikyacu con el turismo, y

$4 \quad$ Recibos de pago es una de las actividades ejecutadas únicamente por la Junta Directiva. Consiste en contabilizar, archivar y declarar los gastos que se usarán en la rendición de cuentas (otra de las actividades) para el reporte que se rinde al programa Bosques.

5 Ropa para venta se ejecuta cuando hay actividades del Comité de Turismo (aplica a otro en Tabla 1-1), y también cuando el comité de artesanas comercializa sus productos, dependiendo los puntos de venta, también venden otros productos adicionales a los elaborados dentro del mecanismo TDC. 
Chunchiwi con las decisiones de la implementación de su cocina comunal, por dar algunos ejemplos.

En los tres sistemas, mutuo, integrador y no complementario, los comuneros cumplen sus diversos roles. Por lo tanto, todos los beneficiarios son individuos interdependientes en diferentes niveles para el logro de sus actividades.

De esta manera, los sistemas se usan para describir estas relaciones, que no son estáticas. Esta investigación no tiene como objetivo clasificar si alguna es mejor que otra. Una pareja (hombre y mujer, mujer y mujer, hombre y hombre), bajo cualquier sistema complementario (mutuo e integrador), puede empoderar al otro.

Finalmente, cabe resaltar que, cuando el acceso a oportunidades es limitado - como lo es en las tres comunidades indígenas de esta investigación-, muchos padres de familia buscan mayores ingresos económicos fuera de la comunidad. Así, su autonomía se ve afectada, así como las bases de su complementariedad de género (Safa, 2005), como evidentemente se da en Chirik Sacha, seguido por Chirikyacu y luego Chunchiwi (muy poco). Los roles de género están cambiando a través de este proceso. En unos años, los sistemas de complementariedad de género pueden ser distintos a los que se han identificado en esta investigación.

\section{Factores que facilitan y limitan la participación de los beneficiarios en el} mecanismo TDC

El mayor aporte de la comunidad a la ejecución del mecanismo es su base de organización comunal. Sin esta, la implementación del programa hubiera sido más complicada. En las tres comunidades, la mayoría de los comuneros reconoce este factor como fundamental para afiliarse al mecanismo.

Desde el inicio del convenio, los comuneros tuvieron diferentes opiniones que fueron cambiando y consolidándose positivamente conforme avanzó la implementación del TDC. Estas opiniones eran conversadas en el hogar. Cabe resaltar que las opiniones variaron por hogar, no por individuo. La tabla 1-5 muestra las opiniones más relevantes por año, según recordaron las familias.

Para el mayor grupo minoritario en la comunidad, los resultados de haberse involucrado en el programa se resumen en beneficios que son aprovechados por ellas mismas, por su comunidad y por sus familias. Ellas reconocen que contribuyen a la implementación del mecanismo, como este a su propio beneficio, forjando un ciclo constante que genera sólidas opiniones (tabla 1-6). 
Tabla 1-5. Perspectivas de los comuneros sobre el TDC en implementación, por año

\begin{tabular}{|c|c|c|c|c|c|}
\hline CCNN & Año 1 & Año 2 & Año 3 & Año 4 & Año 5 \\
\hline \multirow[t]{2}{*}{ Chirikyacu } & $\begin{array}{l}\text { Siempre creyeron } \\
\text { en el programa, } \\
\text { nunca hubo otras } \\
\text { ideas. }\end{array}$ & \multicolumn{4}{|c|}{$\begin{array}{l}\text { Algunos pocos } \\
\text { comuneros, no tan } \\
\text { involucrados creyeron que } \\
\text { había corrupción entre las } \\
\text { autoridades comunales y } \\
\text { con el programa. }\end{array}$} \\
\hline & $\begin{array}{l}\text { Confiaron en el } \\
\text { programa. }\end{array}$ & \multicolumn{4}{|c|}{ Confiaron en el programa. } \\
\hline Chunchiwi & $\begin{array}{l}\text { «Quieren nuestro } \\
\text { territorio» }\end{array}$ & $\begin{array}{l}\text { Confiaron } \\
\text { en el } \\
\text { programa. }\end{array}$ & & & \\
\hline $\begin{array}{l}\text { Chirik } \\
\text { Sacha }\end{array}$ & $\begin{array}{l}\text { «Quieren } \\
\text { el petróleo } \\
\text { de nuestro } \\
\text { territorio». }\end{array}$ & $\begin{array}{l}\text { «Solo se } \\
\text { interesan en } \\
\text { un grupo de } \\
\text { nosotros». }\end{array}$ & $\begin{array}{l}\text { Confiaron } \\
\text { en el } \\
\text { programa. }\end{array}$ & $\begin{array}{l}\text { Confiaron } \\
\text { en el } \\
\text { programa. }\end{array}$ & $\begin{array}{l}\text { Confiaron } \\
\text { en el } \\
\text { programa. }\end{array}$ \\
\hline
\end{tabular}

Para involucrarse en el programa, la motivación fue fundamental. Se caracteriza en motivación propia y motivación por estímulos externos (tabla 1-7). En este caso (testimonio), el estímulo externo proviene de otra participante de su comunidad: «Mi sobrina fue. Ella fue lejos y me contó todo lo que hacían, cuando volvió nos contó y nos interesó todo eso, por eso nos motivó a participar en las mujeres organizadas» (anónimo, CCNN Chirikyacu. Según Schmink y García (2015), en su acotación a Favilla (2006), es posible que la resistencia a participar radique en la posición de sus propias familias, con lo cual concuerdo, porque las conductas aceptadas y no aceptadas no son únicamente familiares, sino también comunales. Esto tiene un gran peso sobre la mujer, y también sobre el varón, y puede llevar a la expulsión de su propia comunidad o al suicidio.

En las mujeres kichwa, es importante el compromiso y la lealtad de palabra. Es también importante cuando la motivación para vincularse en las actividades del programa es propia y no forzada, porque entonces el involucramiento es más duradero y el empoderamiento más sólido. Este punto se evidencia en la constancia del trabajo de las mujeres que actualmente conforman los comités de artesanas: en todas, su motivación principal es propia y su empoderamiento más notorio que en la mayoría de las mujeres que no están involucradas. 
Tabla 1-6. Opinión de la mujer a partir de su experiencia (contribución y beneficio) en el programa Bosques

\begin{tabular}{|c|c|c|}
\hline Contribución & Beneficio & Opinión* \\
\hline $\begin{array}{l}\text { Conocimiento de técnicas } \\
\text { para la elaboración de } \\
\text { artesanías }\end{array}$ & $\begin{array}{l}\text { Adquisición de } \\
\text { capacidades: } \\
\text { Mayor desenvolvimiento en } \\
\text { asociatividad comunal }\end{array}$ & $\begin{array}{l}\text { Se necesitan técnicos } \\
\text { especializados para las otras } \\
\text { actividades (así como en } \\
\text { cacao) }\end{array}$ \\
\hline $\begin{array}{l}\text { Experiencia en el cultivo } \\
\text { de otros cultivos como } \\
\text { el café (aporte para el } \\
\text { cacao) }\end{array}$ & $\begin{array}{l}\text { Perfeccionamiento de } \\
\text { técnicas para elaboración de } \\
\text { chumbis y otros } \\
\text { Mejoramiento de habilidad } \\
\text { para ventas }\end{array}$ & $\begin{array}{l}\text { Se necesita más constancia } \\
\text { en la ejecución de } \\
\text { actividades por parte de } \\
\text { todas las que aceptamos el } \\
\text { compromiso (comuneras) } \\
\text { Los esposos no deben }\end{array}$ \\
\hline $\begin{array}{l}\text { Voluntad y deseo para } \\
\text { aprender }\end{array}$ & $\begin{array}{l}\text { Adquisición de técnicas } \\
\text { de poda, injertación y } \\
\text { abonamiento }\end{array}$ & $\begin{array}{l}\text { fastidiarse si tenemos } \\
\text { que atender más } \\
\text { responsabilidades }\end{array}$ \\
\hline $\begin{array}{l}\text { Dedicación para motivar } \\
\text { a otros en su familia a } \\
\text { que se involucren en el } \\
\text { programa }\end{array}$ & $\begin{array}{l}\text { Conocimiento para armar } \\
\text { almácigos y plantones } \\
\text { Conocimiento de } \\
\text { elaboración y usos del } \\
\text { Bokashi } \\
\text { Aplicación práctica de } \\
\text { conocimiento adquirido }\end{array}$ & $\begin{array}{l}\text { Desde que mi esposo } \\
\text { participa más de las charlas, } \\
\text { hay mejoras en su conducta } \\
\text { en la casa } \\
\text { Es más fácil cultivar cacao } \\
\text { que café }\end{array}$ \\
\hline
\end{tabular}

* La mayoría se colectó en Chirik Sacha.

Según Cervone (2002), entre los grupos indígenas un sistema mantenido de complementariedad de género está basado en su equidad en diversidad, que significa que la diferencia entre el varón y la mujer (que sí existe) «legitima sus capacidades y establece sus derechos para que ambos accedan a las mismas oportunidades» (Safa, 2005). No es el caso en estas comunidades, pues es evidente que las mujeres no acceden a las mismas oportunidades. La tabla 1-7 caracteriza las limitantes en la participación de la mujer por factores internos, que son las minorías comunales, mencionadas repetidas veces en esta investigación. Estas minorías se ven más limitadas si el programa no tiene el propósito de integrarlas. Es notoria la falta de enfoque de género en el planteamiento del programa Bosques, pues en las actividades de involucramiento directo de los varones, la asistencia 
Tabla 1-7. Factores que facilitan y limitan la participación del grupo minoritario principal (mujeres)

\begin{tabular}{|c|c|c|}
\hline \multirow{6}{*}{ 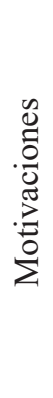 } & $\begin{array}{l}\text { Internos } \\
\text { (motivación propia) }\end{array}$ & $\begin{array}{l}\text { Externos } \\
\text { (motivación a partir de mi entorno) }\end{array}$ \\
\hline & Porque me gusta & Porque a mi esposo le gusta mi trabajo \\
\hline & Porque disfruto de hacerlo & Porque a mi esposo le parece bien \\
\hline & Porque quiero aprender & Porque dicen en la comunidad que tenemos que ir \\
\hline & Porque es importante recibir & Porque otras mujeres nos cuentan cómo es \\
\hline & & Porque la ganancia va a ser buena* \\
\hline \multirow{8}{*}{ 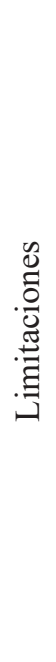 } & $\begin{array}{l}\text { Internos } \\
\text { (minorías comunales) }\end{array}$ & $\begin{array}{l}\text { Externos } \\
\text { (debilitamiento de organización }^{* *} \text { ) }\end{array}$ \\
\hline & $\begin{array}{l}\text { Mujeres con bebés o niños } \\
\text { pequeños }\end{array}$ & $\begin{array}{l}\text { Problemas que surgen dentro de la organización } \\
\text { de mujeres organizadas }\end{array}$ \\
\hline & Mujeres analfabetas & Falta de coordinación sobre gastos adicionales \\
\hline & $\begin{array}{l}\text { Mujeres nuevas en la } \\
\text { comunidad }\end{array}$ & $\begin{array}{l}\text { Falta de confianza entre las miembros del comité } \\
\text { de artesanas }\end{array}$ \\
\hline & Mujeres madres solteras & No participan colectivamente en toma de \\
\hline & Mujeres adultas mayores & $\begin{array}{l}\text { decisiones para contratación adicional de } \\
\text { personal (ajeno a organización) }\end{array}$ \\
\hline & $\begin{array}{l}\text { Mujeres con alguna } \\
\text { discapacidad física }\end{array}$ & Falta de retribución de ganancia equitativamente \\
\hline & & $\begin{array}{l}\text { Falta de equidad en la distribución de trabajo o en } \\
\text { la asignación de funciones }\end{array}$ \\
\hline
\end{tabular}

* Se refiere a cacao. Las demás corresponden a artesanía.

** Problemas identificados en Chirik Sacha.

técnica fue más implementada y bien diseñada para la audiencia de varones - e incluso usó mayores recursos - que en las actividades de involucramiento directo de mujeres, donde aún hace falta capacitación que permita evitar los conflictos externos de la tabla 1-7.

En el caso las mujeres waorani, una de las limitantes para involucrarse en el liderazgo de su comunidad o en actividades que permitieran empoderarla, era la atención a los hijos, y en cuanto a varones, el analfabetismo. Ambos casos aplican en las tres comunidades de esta investigación (tabla 1-7). No obstante, Avilés (2018) encontró que una mujer soltera tiene mayores oportunidades de liderazgo; 
en esta investigación las mujeres solteras en edad adulta son muy pocas y son madres: esto las posiciona como minoría comunal.

\section{CONCLUSIONES}

La complementariedad de género entre los indígenas mayas se ha validado a través de la interdependencia de sus actividades, «proveyendo unidades centrales de importancia simbólica y de funciones, acentuando la dualidad sexual (o paralelismo) y la interrelacionalidad de género; proveyendo dinámicas esenciales del trabajo de la mujer y del varón, y el valor que tiene» (Gero y Scattolin, 2002; Fitzpatrick, 2005). Este no es el caso en ninguna de las tres comunidades kichwa. Las comunidades reconocen las fortalezas que tienen como comunidad, pero no de cada comunero/comunera. El «no reconocimiento» del individuo debilita a largo plazo su asociatividad comunal.

En Chirik Sacha, el rol del técnico de cacao ha sido un elemento clave para la satisfacción de los agricultores y para su vinculación con el programa Bosques. Por lo tanto, es fundamental que, desde la asistencia técnica, se destaque el reconocimiento a cada familia beneficiaria. Es necesario reducir la discriminación dentro de la comunidad y la desconfianza. A continuación, dos testimonios que reafirman la desconfianza: «no les tienen fe a las mujeres con la cría de animales, ellas no saben» (anónimo, CN Chirk Sacha); «aquí en la casa también separo yo la basura. ¿Mi esposo? ¿Él qué va a hacer? Je-je» (anónimo, CN Chirik Sacha).

Entre los hallazgos, destaca el reconocimiento de todos los beneficiarios al programa, pues lo consideran como una oportunidad de aprendizaje, producción, acceso a mercado, y otros: «yo estoy contento porque nunca nadie, ningún programa, nos ha apoyado así, a que aprendamos tanto en todas las parcelas» (anónimo, CN Chirik Sacha).

El reconocimiento es, sin duda, por el ejemplar trabajo al impulsar el componente económico, fortaleciendo el cacao y la artesanía. El cacao generaba mayores ingresos, por lo tanto, se priorizó capacitaciones cacaoteras. Un ejemplo del gran trabajo: «yo pienso que es un técnico muy excelente, él no es egoísta en el campo, él enseña cómo debes hacer tu manejo, cómo debes sembrar tus plantas y cómo mantenerlas, a qué distancia ponerlas, cómo es su tiempo de poda; de todo aprendemos. Todos nosotros recién con el técnico hemos aprendido esto» (anónimo, CN Chirikyacu). El enfoque de las prioridades del PNCB claramente apuntaba a empoderar a los individuos beneficiarios de cacao y el trabajo inicial ha sido realmente bueno para la productividad agrícola (no todavía 
para comercialización, la cual requerirá sumos esfuerzos en el siguiente Plan de Inversión), pero a costa de no tomar en cuenta la estructura comunal - que es familiar - ni de las existentes minorías comunales — que cada vez están más opacadas por el empoderamiento del grupo más favorecido-. Para una visión a corto plazo, las metas sí se van a lograr: un producto artesanal competitivo y una cosecha de cacao que permita mejorar los ingresos familiares, pero se necesita llegar a la meta final: una comercialización sostenible de alto impacto en la economía familiar para reducir la dependencia en los bosques (Ocaña, 2012). Se necesita un sólido enfoque de género en el ámbito familiar en el programa, porque el bajo reconocimiento y la desconfianza entre individuos para cumplir sus responsabilidades del Plan de Inversión, más la falta de oportunidades de las minorías, podrían dejar una notoria brecha al salir del mecanismo TDC. Por lo tanto, el impacto a largo plazo en la comunidad puede ser contraproducente por los roles de género, posicionándose a partir de la intervención del PNCB (que tiene un enfoque de género limitado). Lo preocupante es que los mismos programas, en su etapa de formulación, pueden adelantarse a asumir cuáles son los roles que los comuneros y comuneras deben ejecutar, y más adelante, en la asistencia técnica, perder la oportunidad de capacitar a mujeres o varones, resultando en el fortalecimiento de las inequidades sociales existentes (Pinedo, 2014; Schmink y García, 2015). Por lo tanto, es necesario el entendimiento del enfoque de género antes de solo proponer actividades que cumplan con la cuota de género para mujeres. Si se las va a proponer, es preciso diseñarlas detalladamente para no perjudicar ni a varones ni a mujeres.

El sistema no complementario y de mayor participación directa es dominante entre varones, y los sistemas complementarios (mutua e integradora) son menos dominantes en la implementación del TDC. Los varones jóvenes, padres y bilingües son el grupo poblacional que recibe los mayores beneficios del programa. Los kichwa lamas funcionan desde una base familiar, y en términos comunales, son altamente colaborativos. Con el fin de lograr actividades de interés comunal o familiar, son todos: individuos interdependientes, en diferentes escalas.

El PNCB, desde su diseño inicial, carece de un enfoque de género orientado a comunidades indígenas amazónicas de organización base familiar. Por lo tanto, es fundamental que Bosques-San Martín continúe con las recientes acciones de enfoque de género iniciadas poco después de la fase de campo de esta investigación (2017). Destacan los talleres con enfoque de género para los comités, técnicos y promotores de cacao, y Junta Directiva, con el fin de reiterar el reciente compromiso de involucrar directamente a la mujer en el desarrollo 
de las actividades del TDC. Schmink y García (2015), en su cita a Mello (2014), señalan que las microempresas colectivas - o en este caso, comunitarias - tienen la facilidad de fortalecer su propia toma de decisiones, y para llegar ahí es crucial la consolidación del empoderamiento económico en las capacitaciones que reciben. Por otro lado, si bien a la fecha de esta investigación, las mujeres tenían dos actividades de mayor aprendizaje (artesanía y actividades del vivero), se debe tener sumo cuidado en no dividir lo que deben ejercer la mujer o el varón de lo que no (Avilez, 2008). Enfatizo en este último punto porque sí existen prejuicios respecto de las actividades que deberían ejercer las mujeres en las tres comunidades, más visible en Chirikyacu y Chunchiwi.

Entre los hallazgos, también está el reconocimiento de las mujeres beneficiarias al programa. Todas han admitido alguna de las siguientes tres:

- Todas han admitido Han aprendido en distintos niveles a través del programa.

- Es importante aprovechar las oportunidades de aprendizaje que ofrece el programa Bosques.

- Hay varias formas de aprovechar el aprendizaje, la mayoría de ellas considera que el mayor aprendizaje es para ellas mismas; un segundo grupo afirma que el beneficio del aprendizaje puede ser aplicado a sus hogares, y un tercer grupo opina que este aprendizaje puede ser beneficioso para ser aplicado a la comunidad.

Esta investigación evidencia que el interés de las mujeres en involucrarse es por motivación propia, seguido de influencias externas. Aun cuando solo sea un grupo reducido de mujeres involucradas, ellas influirán a otras mujeres en los cinco años de contrato, así como sucede en los comités de artesanía y como sucedió en el comité de crianza de animales menores (Chirik Sacha). Se debe empoderar a las mujeres involucradas directamente en lugar de aumentar el número de mujeres participantes, al proveer más experiencias en las actividades de involucramiento directo - como lo hicieron en artesanía-, pues esto les da mayor visibilidad (Avilés, 2008), y los talleres de capacitación en microfinanciamiento, venta de producto o afines deben incluir segmentos de liderazgo y empoderamiento (Pinedo, 2014, en su cita a Mayoux, 2001) para sus actividades dentro y fuera de la comunidad. Cuando ellas tienen la oportunidad de intercambiar conocimiento en actividades fuera de la comunidad, las familias fortalecen sus vínculos, porque los roles de género empiezan a variar (no aplica a todos los casos), evidenciando que la complementariedad de género no es estática y que las normas de género no son las únicas que la definen. Los tres sistemas 
de complementariedad de género identificados en esta investigación enmarcan roles de género (presentes ahora), y que pueden cambiar en el futuro, mientras más sistemas sean desarrollados o adoptados. Por ejemplo, las experiencias de la mujer fuera de la comunidad en capacitaciones de Bosques y en ferias resulta en una apertura temporal para desarrollar nuevos roles de género que en el futuro pueden crear nuevas normas de género.

Finalmente, después de la experiencia de participación de estas tres comunidades en el programa, se confirma que para una efectiva implementación del TDC, Bosques debe continuar con el modelo de cinco años de implementación. Tres años no son suficientes para lograr la participación y el progreso en los diferentes aspectos que requiere cada comunidad: (i) tiempo para entender cómo funciona el programa (para los comuneros); (ii) tiempo para ganar la confianza de la comunidad entera; (iii) tiempo para esperar los resultados de los comités promovidos por el programa Bosques (e.g.: turismo, artesanía, cacao, cuyes, pollos, biohuertos); (iv) aprendizaje constante durante la implementación del mecanismo TDC, que necesita constancia para consolidarse, y (v) una producción agrícola que no puede ser apresurada: en tres años de contrato, hay menos de la mitad de cacao cosechado por agricultores - comparado a las cosechas en un contrato de cinco años-, porque los plantones y el crecimiento de la planta (para producir) por zonas en cada parcela tardan más de un año. Adicionalmente, cada grupo indígena es un mundo distinto. Cabe destacar que las tres comunidades kichwa lamas tienen amplia experiencia implementando proyectos y trabajando con programas de desarrollo rural. Tres años de contrato para comunidades más remotas con menor experiencia son inviables.

\section{Agradecimientos}

Esta investigación se realizó gracias al apoyo de la Universidad de Florida, GIZPerú, Programa Bosques - zonal de San Martín y comunidades beneficiarias del programa.

\section{REFERENCIAS BIBLIOGRÁFICAS}

Aviles, Mayra (2008). Narratives of resistance: An ethnographic view of the emergence of the Huaorani women's association in the Ecuadorian Amazon. Gainesville: University of Florida. 
Bosques (2016). [Programa Nacional de Conservación de Bosques para la Mitigación del Cambio Climático]. www.bosques.gob.pe

Fitzpatrick, Susan. (2005). Gender and Religion: Gender and South American Religions. Encyclopedia of Religion. Recuperado de https:/www.encyclopedia.com/ environment/encyclopedias-almanacs-transcripts-and-maps/gender-and-religion-gender-and-south-american-religions

Gero, Joan M. y Scattolin, M.Cristina. (2002). Beyond Complementarity and Hierarchy: New Definitions for Archaeological Gender Relations. In Pursuit of Gender (pp. 155-171).

Ministerio del Ambiente (2016). Plan de acción en género y cambio climático. Lima: Minam.

Ocaña, Stephanie. (2012). El papel de los bosques en la disminución de la pobreza y la desigualdad evidencia de la Amazonía peruana. México, D.F.: Centro de Investigación y Docencia Económicas (CIDE).

Paulson, Susan. (2000). La diferencia e interdependencia social en el manejo agroforestal. Agroforestería en las Américas, 7(25).

Pinedo, Dulhy. (2014). The politics of sociality: Social networks and indigenous mobilization in the Peruvian Amazon. Gainesville: Universidad de Florida.

Proyecto CBC (2013). Conservando bosques comunitarios en el Perú: sistematización del proyecto Conservación de Bosques Comunitarios. Lima: GIZ, BMU, MINAM, Bosques, Gobierno del Perú.

Ruedas, Javier. (2003). Gender and Sociality in Amazonia: How Real People are Made. Tipiti: Journal of the Society for the Anthropology of Lowland South America, Vol 1: Iss. 1, Article 11.

Safa, Helen. (2005). Challenging Mestizaje. A Gender Perspective on Indigenous and Afrodescendant Movements in Latin America. Critique of Anthropology, 25(3), 307-330.

Schmink, Marianne y García, Marliz. (2015). Bajo el dosel: género y bosques en la Amazonía. Documentos Ocasionales 125. Bogor: CIFOR.

United States Agency for International Development - USAID (2014). Diagnóstico de género en la Amazonía, Amazonas, Loreto, Madre de Dios, San Martín y Ucayali. Programa Pro Descentralización. Lima: CIAM, Ministerio de la Mujer y de Poblaciones Vulnerables, del Perú.

Van Penny. (2000). Materializing Thailand Thai Genders and the Limits of Western Gender Theory. Intersections: Gender, History and Culture in the Asian Context, $X(10)$, XI-274. 